\title{
Otto Vejvoda
}

On the periodic solution of a quasi-linear non-autonomous system

Czechoslovak Mathematical Journal, Vol. 11 (1961), No. 1, 62-75

Persistent URL: http://dml.cz/dmlcz/100443

\section{Terms of use:}

(C) Institute of Mathematics AS CR, 1961

Institute of Mathematics of the Czech Academy of Sciences provides access to digitized documents strictly for personal use. Each copy of any part of this document must contain these Terms of use.

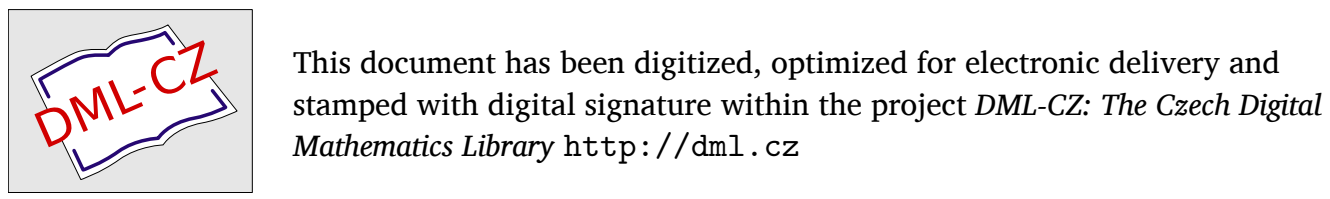




\title{
ON THE PERIODIC SOLUTION OF A QUASI-LINEAR NON-AUTONOMOUS SYSTEM
}

\author{
Otтo Vejvoda, Praha
}

(Received October 2, 1959)

The main purpose of this paper is to appraise the range of a small parameter $\varepsilon$ for which a periodic solution of $(0 \cdot 1)$ exists.

Let us consider the real system

$$
\dot{x}=A x+\varepsilon f(t, x, \varepsilon),
$$

where $\mathbf{x}$ is a $n$-dimensional column vector with components $\left(x_{1}, x_{2}, \ldots, x_{n}\right), \boldsymbol{A}$ is a $n \times n$ constant matrix and $\mathbf{f}(t, \mathbf{x}, \varepsilon)$ is a $n$-dimensional vector function which, besides fulfilling certain continuity and differentiability conditions, is periodic in $t$ of period $2 \pi$ (we shall now shorten to $2 \pi$-periodic), whereas $\frac{\partial f}{\partial t}(t, \mathbf{x}, \varepsilon) \neq 0$. A real positive number $\varepsilon$ is a so-called small parameter.

In several recent papers the question has been studied how large a number $\varepsilon^{*}>0$ we can choose, so that for all $\varepsilon \in\left(0, \varepsilon^{*}\right\rangle$ the existence of a $2 \pi$-periodic solution of $(0.1)$ would be guaranteed. We seek only such $2 \pi$-periodic solutions which for $\varepsilon \rightarrow 0$ tend to a $2 \pi$-periodic solution of

$$
\dot{y}=A y .
$$

A. A. Kruming [1] and A. E. Gel'man [3] suppose that (1) matrix $\boldsymbol{A}$ has no characteristic root of the form $p i$ ( $p$ some integer including zero) and (2) the function $\boldsymbol{f}(t, \boldsymbol{x}, \varepsilon)$ is continuous in $t$ and analytic in $\boldsymbol{x}$ and $\varepsilon$. Ju. A. RJABov [4] retains only assumption (2).

In D. C. LewIs's papers [5], [6] the system

$$
\dot{\boldsymbol{x}}=\boldsymbol{A}(t, \varepsilon) \mathbf{x}+\boldsymbol{F}(t, \mathbf{x}, \varepsilon),
$$

where the matrix $\boldsymbol{A}(t, \varepsilon)$ and the function $\boldsymbol{F}(t, \mathbf{x}, \varepsilon)$ are $2 \pi$-periodic in $t$, is dealt with. If we carry out a transformation of variables so that the coefficients at linear members in $\mathbf{x}$ are constants (such a transformation always exists) then in paper [5] the case is discussed in which condition (1) is fulfilled and further $F_{j}(t, 0,0)=\frac{\partial F_{j}}{\partial x_{k}}(t, 0,0)=0$ 
$(j, k=1,2, \ldots, n)$. In paper [6] the assumption (1) is omitted and the function $\boldsymbol{F}(t, \mathbf{x}, \varepsilon)$ is assumed to be of class $C^{2}$ in $\mathbf{x}$ and $\varepsilon$, further to fulfil

$$
F_{j}(t, 0,0)=\frac{\partial F_{j}}{\partial x_{k}}(t, 0,0)=\frac{\partial^{2} F_{j}}{\partial x_{k} \partial x_{l}}(t, 0,0)=0(j, k, l=1,2, \ldots, n)
$$

and finally to fulfil some other conditions which are less important and more involved. By means of a Green matrix, constructed for the system $\dot{\mathbf{y}}=\boldsymbol{A}(t, \varepsilon) \mathbf{y}$ with periodic boundary conditions, Lewis reduces the problem to the study of a certain system of integral equations.

For an autonomous system (i. e. $\left.\frac{\partial f}{\partial t} \equiv 0\right)$ J. KURzWEIL [7] published an estimate of the interval $\left(0, \varepsilon^{*}\right\rangle$ under very general assumptions (he uses the method of succesive approximations in a similar way as I. G. MALKIN).

In this paper the estimation of the interval $\left(0, \varepsilon^{*}\right\rangle$, for whose values the $2 \pi$-periodic solution exists, is made for the resonant and nonresonant cases (i. e. assumption (1) being or not being fulfilled) under more general assumptions than in papers [1] - [6], because I only suppose that the function $f(t, x, \varepsilon)$ is of class $C^{1}$ in $\boldsymbol{x}$ and of class $C^{0}$ in $\varepsilon$ (similarly as in Kurzweil's paper [7]). My investigation is based mainly on the Coddington-Levinson existence theorem (cf. [8]).

\section{SOME NOTATIONS AND DEFINITIONS}

Let $\boldsymbol{C}$ be a $n \times n$ matrix $\left(c_{i j}\right)(i, j=1,2, \ldots, n)$. Let $\mathscr{N}$ denote the naturally ordered set $\{1,2, \ldots, n\}$, Let $\mathscr{A}$ and $\mathscr{B}$ be naturally ordered subsets of $\mathscr{N}, \mathscr{A}=\left\{a_{1}, a_{2}, \ldots, a_{p}\right\}$, $\mathscr{B}=\left\{b_{1}, b_{2}, \ldots, b_{q}\right\}$, (so that $1 \leqq a_{1}<a_{2}<\ldots<a_{p} \leqq n, 1 \leqq b_{1}<b_{2}<\ldots<$ $\left.<b_{q} \leqq n\right)$. Let us introduce the notation

$$
\boldsymbol{C}_{\mathscr{A} \mathscr{B}}=\left(c_{p q}\right), \quad p \in \mathscr{A}, q \in \mathscr{B} .
$$

(If $\mathscr{A}$ or $\mathscr{B}$ is the empty set then $C_{\mathscr{A} \mathscr{B}}$ has of course no meaning.)

Further, let us denote by $\overline{\mathscr{A}}$ the complement of $\mathscr{A}$ with respect to $\mathscr{N}$ (again naturally ordered).

Analogously, $\mathbf{x}$ being a $n$-vector, $\boldsymbol{x}_{\mathscr{A}}$ denotes the vector $\left(x_{j}\right), j \in \mathscr{A}$.

The symbol + is defined by the relation

$$
c=c_{\mathscr{A}}+c_{\overline{\mathscr{A}}}
$$

where $\boldsymbol{c}$ is a $n$-vector.

We define the norm of a $m \times n$ matrix $C$, whose elements are continuous functions of $t$ on $\langle a, b\rangle$ as

$$
\|\boldsymbol{C}\|=\max _{a \leqq t \leqq b} \max _{k} \sum_{j=1}^{m}\left|c_{j k}(t)\right| .
$$


The norm of a column $n$-vector whose elements are continuous functions of $t$ on $\langle a, b\rangle$ is defined as

$$
\|\mathbf{x}\|=\max _{a \leqq t \leqq b} \sum_{j=1}^{n}\left|x_{j}(t)\right| .
$$

Clearly, the following relations hold,

$$
\|A+B\| \leqq\|A\|+\|B\|, \quad\|A B\| \leqq\|A\|\|B\|, \quad\|A x\| \leqq\|A\|\|x\| .
$$

$\boldsymbol{E}_{n}$ will denote the $n$-dimensional unit matrix. (If no danger of confusion will arise, the index $n$ will be omitted).

We shall say that a real $n \times n$ matrix $A$ is in a semi-canonical form if it is of the form

$$
A=\left(\begin{array}{llll}
\boldsymbol{A}_{1} \cdot \cdot A_{k} B_{1} & & \\
& & \cdot & \\
& & B_{m} & C
\end{array}\right]
$$

where $\boldsymbol{A}_{j}, j=1,2, \ldots, k$ are of the form

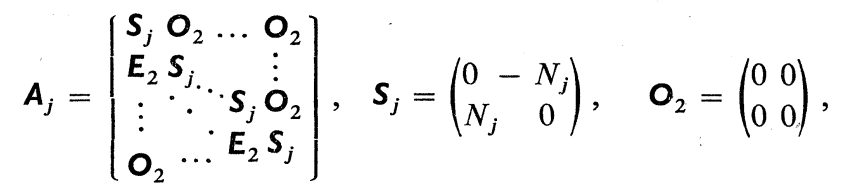

( $N_{j}$ being a positive integer), $\mathbf{B}_{l}, l=1,2, \ldots, m$ are of the form

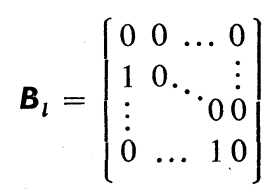

and the matrix $C$ has no characteristic root of the form $p i, p$ being any integer including 0 .

It holds (cf., e. g. [8])

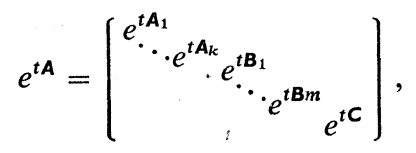

where (the matrix $\boldsymbol{A}_{j}$ being of the type $\alpha_{j} \times \alpha_{j}, \alpha_{j}=2 p_{j}$ )

$(0.6) \quad e^{t \boldsymbol{A}_{j}}=\left(\begin{array}{cccc}e^{t \boldsymbol{S}_{j}} & \mathbf{O}_{2} & \ldots & \mathbf{O}_{2} \\ \frac{t}{1 !} e^{t \boldsymbol{S}_{j}} & e^{t \boldsymbol{S}_{j}} & \ldots & \mathbf{O}_{2} \\ \vdots & & & \\ \frac{t^{p_{j-1}}}{\left(p_{j}-1\right) !} e^{t \boldsymbol{S}_{j}} & \frac{t^{p_{j-2}}}{\left(p_{j}-2\right) !} e^{t \boldsymbol{S}_{j}} \ldots & e^{t \boldsymbol{S}_{j}}\end{array}\right], \quad e^{t \boldsymbol{S}_{j}}=\left(\begin{array}{ll}\cos N_{j} t-\sin N_{j} t \\ \sin N_{j} t & \cos N_{j} t\end{array}\right)$ 
and $\left(\boldsymbol{B}_{l}\right.$ being of the type $\left.\beta_{l} \times \beta_{l}\right)$

$$
e^{t \mathbf{B}_{l}}=\left[\begin{array}{cccc}
1 & 0 & \ldots & 0 \\
t & 1 & \ldots & 0 \\
\frac{t^{\beta_{l}-1}}{\left(\beta_{l}-1\right) !} & \frac{t^{\beta_{l}-2}}{\left(\beta_{l}-2\right) !} & \ldots & 1
\end{array}\right]
$$

In particular, it holds

$$
e^{2 \pi A_{j}}=\left(\begin{array}{cccc}
E_{2} & O_{2} & \ldots & O_{2} \\
\frac{2 \pi}{1 !} E_{2} & E_{2} & \ldots & O_{2} \\
\vdots & & & \\
\frac{(2 \pi)^{p_{j}-1}}{\left(p_{j}-1\right) !} & E_{2} \frac{(2 \pi)^{p_{j}-2}}{\left(p_{j}-2\right) !} & \ldots & E_{2}
\end{array}\right), \quad O_{2}=\left|\begin{array}{ll}
0 & 0 \\
0 & 0
\end{array}\right| .
$$

Let the matrices $\boldsymbol{A}, \boldsymbol{A}_{j}, \boldsymbol{B}_{l}$ and $\boldsymbol{C}$ fulfil all assumptions as above. Then the indices

$$
\begin{gathered}
1,2, \alpha_{1}+1, \alpha_{1}+2, \ldots, \sum_{j=1}^{k-1} \alpha_{j}+1, \sum_{j=1}^{k-1} \alpha_{j}+2, \sum_{j=1}^{k} \alpha_{j}+1, \\
\sum_{j=1}^{k} \alpha_{j}+\beta_{1}+1, \ldots, \sum_{j=1}^{k} \alpha_{j}+\sum_{l=1}^{m-1} \beta_{l}+1
\end{gathered}
$$

will be called singular indices and the (naturally) ordered set formed by them will be denoted $\mathscr{S}$.

The indices

$$
\alpha_{1}-1, \alpha_{1} \ldots, \sum_{j=1}^{k} \alpha_{j}-1, \sum_{j=1}^{k} \alpha_{j}, \sum_{j=1}^{k} \alpha_{j}+\beta_{1}, \ldots, \sum_{j=1}^{k} \alpha_{j}+\sum_{l=1}^{m} \beta_{l}
$$

will be called exceptional indices and the (naturally) ordered set formed by them will be denoted $\mathscr{V}$.

Let the vector function $\boldsymbol{f}(t, \boldsymbol{x}, \varepsilon)$ be defined for $0 \leqq t \leqq 2 \pi, \boldsymbol{x} \in \mathfrak{X}$ and $0 \leqq \varepsilon \leqq \varepsilon_{0}$, where $\mathfrak{X}=E\left[\mathbf{x} \mid\left\|\boldsymbol{x}-\mathbf{x}_{0}\right\|<\rho, \rho>0\right]$. We shall say that $\boldsymbol{f}(t, \boldsymbol{x}, \varepsilon)$ is of class $C^{0}$ in $t$, of class $C^{p}$ in $\boldsymbol{x}$ and of class $C^{r}$ in $\varepsilon$ on $\mathfrak{M}=\langle 0,2 \pi\rangle \times \mathfrak{X} \times\left\langle 0, \varepsilon_{0}\right\rangle$ if it is continuous in $t$ and has continuous partial derivatives of order $p$ in $\mathbf{x}$ and of order $r$ in $\varepsilon$ for every $(t, \mathbf{x}, \varepsilon) \in \mathfrak{M}$. If the derivatives of the highest order $q$ in $\mathbf{x}$ fulfil a Lipschitz condition with respect to $\mathbf{x}$, we shall say that $\boldsymbol{f}(t, \boldsymbol{x}, \varepsilon)$ is of order $C^{q L}$. Similarly in analogous cases of other variables.

A $m$-dimensional vector function $\boldsymbol{h}(\boldsymbol{c}, \varepsilon)$ of an $n$-vector $\boldsymbol{c}$ and a scalar $\varepsilon$ being of class $C^{p}, p \geqq 1$, in $\boldsymbol{c}$ for $c \in \mathfrak{R}, \varepsilon \in \mathbb{E}$ (where $\mathfrak{R}$ is an open set) we shall denote

$$
\frac{\mathrm{Dh}}{\mathbf{D} \boldsymbol{c}}(\mathbf{c}, \varepsilon) \equiv \boldsymbol{h}_{\mathbf{c}}(\mathbf{c}, \varepsilon)=\left(\frac{\partial \boldsymbol{h}_{j}}{\partial \mathbf{c}_{k}}(\mathbf{c}, \varepsilon)\right) \quad(j=1,2, \ldots, m ; k=1,2, \ldots, n)
$$

Given a function $\boldsymbol{f}(t, \mathbf{x}, \varepsilon)$ defined for $0 \leqq t \leqq 2 \pi, \mathbf{x} \in \mathfrak{X}=E\left[\mathbf{x} \mid\left\|\mathbf{x}-\mathbf{x}_{0}\right\|<\rho\right.$, $\rho>0], 0 \leqq \varepsilon \leqq \varepsilon_{0}$ and of class $C^{1}$ in $\boldsymbol{x}$, let us recall the Theorem of the Mean,

$$
\boldsymbol{f}\left(t, \mathbf{x}^{\prime}, \varepsilon\right)-\boldsymbol{f}\left(t, \mathbf{x}^{\prime \prime}, \varepsilon\right)=\int_{0}^{1} \boldsymbol{f}_{\mathbf{x}}\left(t, \mathbf{x}^{\prime \prime}+\vartheta\left(\mathbf{x}^{\prime \prime}-\mathbf{x}^{\prime}\right), \varepsilon\right) \mathrm{d} \vartheta .\left(\mathbf{x}^{\prime}-\mathbf{x}^{\prime \prime}\right) .
$$




\section{THE LOCAL EXISTENCE THEOREM AND THE ESTIMATION OF THE INTERVAL OF EXISTENCE}

Let us consider the (real) differential system

$$
\dot{\mathbf{x}}=\boldsymbol{A x}+\varepsilon f(t, \mathbf{x}, \varepsilon)
$$

where $\mathbf{x}$ is a column $n$-vector $\left(x_{1}, x_{2}, \ldots, x_{n}\right), \boldsymbol{f}$ is a $n$-dimensional vector function, $\boldsymbol{A}$ is a $n \times n$ constant matrix and $\varepsilon>0$ is a small parameter. Without loss of generality we may suppose that $\boldsymbol{A}$ has a semi-canonical form.

We will prove next the following theorem:

Theorem 1.1. Let the following assumptions be fulfilled.

(I) The system of equations

$$
\begin{aligned}
& \boldsymbol{H}_{\mathscr{\mathscr { S }}}^{(0)} \equiv\left(e^{2 \pi \boldsymbol{A}}-\boldsymbol{E}\right)_{\overline{\mathscr{S}} \overline{\mathcal{V}}} \boldsymbol{c}_{\mathscr{V}}^{(0)}=0, \\
& \boldsymbol{H}_{\mathscr{S}}^{(0)} \equiv \int_{0}^{2 \pi} e_{\mathscr{\mathscr { S }} \mathcal{S}}^{(2 \pi-s) \boldsymbol{A}} \mathrm{f}\left(s, e^{s \boldsymbol{A}} \boldsymbol{c}^{(0)}, 0\right) \mathrm{d} s=0
\end{aligned}
$$

has a real solution $\mathrm{c}^{(0)}=\mathrm{c}^{(0) *}$.

(II) Given $\varepsilon_{0}>0$ and $\rho_{0}>0$, let us denote

$$
\mathfrak{X}_{\rho_{0}}=E\left[\mathbf{x} \mid\left\|\mathbf{x}-e^{t \boldsymbol{A}} \mathbf{c}^{(0) *}\right\|<\rho_{0} \text { for } 0 \leqq t \leqq 2 \pi\right]
$$

and $\mathfrak{M}=E\left[t, \mathbf{x}, \varepsilon \mid 0 \leqq t \leqq 2 \pi, \mathbf{x} \in \mathfrak{X}_{\rho_{0}}, 0 \leqq \varepsilon \leqq \varepsilon_{0}\right]$. The function $\boldsymbol{f}$ is on $\mathfrak{M}$ $2 \pi$-periodic in $t$, of class $C^{0}$ in $t$ and $\varepsilon$ and of class $C^{1}$ in $\mathbf{x}$.

(III) The Jacobian of the system $(1.2,1.3)$ with respect to $c^{(0)}$ at the point $c^{(0) *}$ is nonvanishing.

(IV) Let $\varepsilon^{*}=\min \left(\varepsilon_{1}, \varepsilon_{2}, \varepsilon_{3}\right)$, where the $\varepsilon_{i}$ are defined by equations (1.33), (1.35) and (1.44).

Then, for every $\varepsilon$ from the interval $\left(0, \varepsilon^{*}\right\rangle$ there exists a unique $2 \pi$-periodic solution $\phi\left(t, c^{*}(\varepsilon), \varepsilon\right)$ of the system (1.1) with the initial condition $\phi\left(0, c^{*}(\varepsilon), \varepsilon\right)=c^{*}(\varepsilon) \in C^{0}$. This solution tends for $\varepsilon \rightarrow 0$ to the $2 \pi$-periodic solution $e^{t A} c^{(0)^{*}}$ of the system

$$
\dot{\mathbf{y}}=\mathbf{A y} \text {. }
$$

Proof. By the assumption (II) and by the theorem on the continuous dependence of a solution on initial conditions and on parameters there exist numbers $\bar{\varepsilon}_{0}, 0<\bar{\varepsilon}_{0} \leqq$ $\leqq \varepsilon_{0}$ and $\mu_{0}, 0<\mu_{0} \leqq \rho_{0}$ such that every solution $\psi(t, \mathbf{c}, \varepsilon)$ of the system (1.1), in which $0<\varepsilon \leqq \bar{\varepsilon}_{0}$, satisfying the initial conditions $\psi(0, \boldsymbol{c}, \varepsilon)=\boldsymbol{c}$, where $\boldsymbol{c} \in \mathfrak{l}_{\mu_{0}}=$ $=E\left[\mathbf{c} \mid\left\|\mathbf{c}-\mathbf{c}^{0 *}\right\| \leqq \mu_{0}\right]$, exists on the interval $\langle 0,2 \pi\rangle$ and

$$
\psi(t, c, \varepsilon) \in \mathfrak{X}_{\rho_{0}} \text { for } 0 \leqq t \leqq 2 \pi, c \in \mathfrak{R}_{\mu_{0}}, 0 \leqq \varepsilon \leqq \bar{\varepsilon}_{0}
$$

(cf. [8], chap. 2, th. 4.3).

Also by the assumption (II), solutions of the system (1.1) are uniquely determined in $\mathfrak{X}_{\rho_{0}}$ by the initial conditions. From the variation-of-constants method it follows that the system (1.1) is equivalent to the system of integral equations

$$
\mathbf{X}=e^{t \boldsymbol{A}} \boldsymbol{c}+\varepsilon \int_{0}^{t} e^{(t-s) A} f(s, \mathbf{x}, \varepsilon) \mathrm{d} s,
$$


i. e. every solution of (1.1) with initial condition $\mathbf{x}(0)=\boldsymbol{c}$ satisfies (1.6) and vice versa.

According to the uniqueness of the solutions for $0 \leqq \varepsilon \leqq \bar{\varepsilon}_{0}$ and $c \in \mathfrak{R}_{\mu_{0}}$, a necessary and sufficient condition for a solution $\phi(t, c, \varepsilon)$ of (1.6) (or (1.1)) with initial value $\phi(0, c, \varepsilon)=c$ to be $2 \pi$-periodic, is that

(1.7) $\phi(2 \pi, c, \varepsilon)-\phi(0, c, \varepsilon) \equiv\left(e^{2 \pi \boldsymbol{A}}-E\right) c+\varepsilon \int_{0}^{2 \pi} e^{(2 \pi-s) A} f(s, \phi(s, c, \varepsilon), \varepsilon) \mathrm{d} s=0$.

It is now our task to find under which conditions and for how large $\varepsilon$ an initial vector $c=c^{*}(\varepsilon)$ exists such that the conditions (1.7) are fulfilled and $c^{*}(0)=c^{(0) *}$.

If the matrix $\boldsymbol{A}$ has no characteristic root of the type $p i$ ( $p$ being an integer including 0), i. e. we have a non-resonant case, then $\operatorname{det}\left(e^{2 \pi \boldsymbol{A}}-\boldsymbol{E}\right)=\operatorname{det}\left(e^{2 \pi \boldsymbol{C}}-\mathbf{E}\right) \neq 0$ and the system (1.7) need not be modified. In the opposite case the matrix $\boldsymbol{A}$ has at least one sub-matrix of the type $\boldsymbol{A}_{j}$ or $\boldsymbol{B}_{l}$ and then, with respect to (0.7), (0.8) det $\left(e^{2 \pi \boldsymbol{A}}-\boldsymbol{E}\right)=0$. Hence, for $\varepsilon=0$ the system (1.7) does not determine the vector $c^{*}(0)$ uniquely and therefore we cannot use the implicit function theorem to prove the existence of a solution of (1.7) for $\varepsilon>0$.

Using relations $(0.7)$ and $(0.8)$ we find easily

$$
\left(e^{2 \pi \boldsymbol{A}}-\boldsymbol{E}\right)_{\mathscr{S} \mathcal{H}}=0,\left(e^{2 \pi \boldsymbol{A}}-\boldsymbol{E}\right)_{\mathcal{N} \mathscr{V}}=0, \operatorname{det}\left(e^{2 \pi \boldsymbol{A}}-\boldsymbol{E}\right)_{\overline{\mathscr{S}} \overrightarrow{\mathcal{V}}} \neq 0 .
$$

Hence, we can write the system (1.7) in the form

$$
\begin{aligned}
& \boldsymbol{H}_{\overline{\mathscr{S}}} \equiv\left(e^{2 \pi \boldsymbol{A}}-\boldsymbol{E}\right)_{\overline{\mathscr{S}} \overline{\mathcal{V}}} \boldsymbol{c}_{\overline{\mathcal{V}}}+\varepsilon \int_{0}^{2 \pi} e_{\overline{\mathscr{S} \mathcal{S}}}^{(2 \pi-s) A} f(s, \phi, \varepsilon) \mathrm{d} s=0, \\
& \boldsymbol{H}_{\mathscr{S}} \equiv \int_{0}^{2 \pi} e_{\mathscr{S} \mathcal{S}}^{(2 \pi-s) A} f(s, \phi, \varepsilon) \mathrm{d} s=0 .
\end{aligned}
$$

(We might divide the equations (1.10) by $\varepsilon$ because of $\varepsilon \neq 0$ ).

The left sides of (1.9) and (1.10) are uniformly continuous in $c$ and $\varepsilon$ for $c \in \mathfrak{R}_{\mu_{0}}$ and $0 \leqq \varepsilon \leqq \bar{\varepsilon}_{0}$ and they reduce for $\varepsilon=0$ (in accordance with (1.6)) to the systems (1.2) and (1.3).

Let us make some small modifications in (1.9) and (1.10). Let us denote

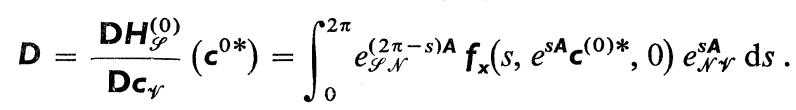

By (III) det $\boldsymbol{D} \neq 0$ and therefore the inverse matrix $\boldsymbol{D}^{-1}$ exists. By (1.8) there exists the inverse matrix $\left(e^{2 \pi \boldsymbol{A}}-\mathbf{E}\right)^{\frac{-1}{\mathcal{S}} \tilde{V}}=\boldsymbol{C}$. Hence, the equations (1.9) and (1.10) are evidently equivalent to the equations

$$
\begin{aligned}
& \boldsymbol{c}_{\mathscr{V}}=-\varepsilon \boldsymbol{C} \int_{0}^{2 \pi} e_{\overline{\mathscr{G}} \mathcal{V}}^{(2 \pi-s) A} f(s, \phi(s), \varepsilon) \mathrm{d} s, \\
& \boldsymbol{c}_{\mathscr{V}}=\boldsymbol{c}_{\mathscr{V}}-\mathbf{D}^{-1} \int_{0}^{2 \pi} e_{\mathscr{\mathcal { S }}}^{(2 \pi-s) A} f(s, \phi(s), \varepsilon) \mathrm{d} s .
\end{aligned}
$$

To prove our theorem we use the method of successive approximations. We define

$$
\begin{aligned}
& c_{0, \overline{\mathscr{V}}}=c_{\mathscr{V}}^{(0) *}=0, \\
& c_{0, \mathscr{V}}=c_{\mathscr{V}}^{(0) *}, \\
& \phi_{0}(t)=e^{t A} c_{0},
\end{aligned}
$$




$$
\begin{aligned}
& \boldsymbol{c}_{k+1, \bar{\gamma}}=-\varepsilon \boldsymbol{C} \int_{0}^{2 \pi} e_{\overline{\mathscr{S}} \mathcal{N}}^{(2 \pi-s) \mathrm{A}} \mathrm{f}\left(s, \phi_{k}(s), \varepsilon\right) \mathrm{d} s, \\
& \boldsymbol{c}_{k+1, \mathscr{V}}=\boldsymbol{c}_{k, \mathfrak{V}}-\boldsymbol{D}^{-1} \int_{0}^{2 \pi} e_{\mathscr{S}_{\mathcal{S}}}^{(2 \pi-s) \boldsymbol{A}} f\left(s, \phi_{k}(s), \varepsilon\right) \mathrm{d} s, \\
& \phi_{k+1}(t)=e^{t \boldsymbol{A}} \boldsymbol{c}_{k+1}+\varepsilon \int_{0}^{t} e^{(t-s) A} f\left(s, \phi_{k}(s), \varepsilon\right) \mathrm{d} s .
\end{aligned}
$$

In the estimations below, the following assumptions will be supposed to be fulfilled:

$$
\begin{aligned}
& \left(P_{1}\right) 0 \leqq \varepsilon \leqq \bar{\varepsilon}_{0}, \quad 0 \leqq t \leqq 2 \pi, \\
& \left(P_{2}\right) c_{k} \in \mathfrak{l}_{\mu}, \quad 0<\mu \leqq \mu_{0}, \\
& \left(P_{3}\right) \phi_{k}(t) \in \mathfrak{X}_{\rho}, \quad 0<\rho<\rho_{0} .
\end{aligned}
$$

Clearly, there exist constants $a, b_{1}, b_{0}, b, c_{1}, c_{2}, c$, and $c_{0}$ such that

(1.13) $\left\|e^{t \mathrm{~A}}\right\| \leqq a$,

(1.14) $\left\|C \int_{0}^{2 \pi} e_{\mathscr{\mathcal { S }} \mathcal{S}}^{(2 \pi-s) \mathrm{A}} \mathrm{f}\left(s, \phi_{0}(s), \varepsilon\right) \mathrm{d} s\right\| \leqq b_{1}$,

(1.15) $\left\|\int_{0}^{t} e^{(t-s) A} f\left(s, \phi_{0}(s), \varepsilon\right) \mathrm{d} s\right\| \leqq b_{0}$,

(1.16) $\left\|\int_{0}^{t} e^{(t-s) A} f\left(s, \phi_{k}(s), \varepsilon\right) \mathrm{d} s\right\| \leqq b \quad(k=1,2, \ldots)$,

(1.17) $\left\|C \int_{0}^{2 \pi} e_{\mathcal{G}_{\mathcal{H}}}^{(2 \pi) \mathrm{A}} \int_{0}^{1} f_{\mathbf{x}}\left(s, \phi_{k-1}+\vartheta\left(\phi_{k}-\phi_{k-1}\right), \varepsilon\right) \mathrm{d} \vartheta \mathrm{d} s\right\| \leqq c_{1}$,

(1.18) $\| \boldsymbol{D}^{-1} \int_{0}^{2 \pi} e_{\mathcal{S} \mathcal{S}}^{(2 \pi-s) A} f_{\mathbf{x}}\left(s, \phi_{0}, 0\right)\left[0+(-1) e_{\mathcal{N} \bar{Y}}^{s A} C \int_{0}^{2 \pi} e_{\mathcal{S}_{\mathcal{S}}}^{(2 \pi-\sigma) A} f\left(\sigma, \phi_{0}, \varepsilon\right) \mathrm{d} \sigma+\right.$ $\left.+\int_{0}^{s} e^{(s-\sigma) A} f\left(\sigma, \phi_{0}, \varepsilon\right) \mathrm{d} \sigma\right] \mathrm{d} s \| \leqq c_{0}$,

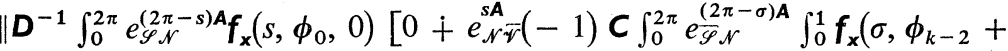

$$
\begin{aligned}
& \left.+\vartheta\left(\phi_{k-1}-\phi_{k-2}\right), \varepsilon\right) \mathrm{d} \vartheta \mathrm{d} \sigma+D^{-1} \int_{0}^{2 \pi} e_{\mathscr{S}_{\mathcal{S}}}^{(2 \pi-s) A} f_{\mathbf{x}}\left(s, \phi_{0}, 0\right) \int_{0}^{s} e^{(s-\sigma) A} \text {. }
\end{aligned}
$$$$
\text { . } \left.\int_{0}^{1} f_{\mathbf{x}}\left(\sigma, \phi_{k-2}+\vartheta\left(\phi_{k-1}-\phi_{k-2}\right), \varepsilon\right) \mathrm{d} \vartheta \mathrm{d} \sigma\right] \mathrm{d} s \| \leqq c_{2},
$$

(1.20) $\left\|\int_{0}^{t} e^{(t-s) \mathbf{A}} \int_{0}^{1} f_{\mathbf{x}}\left(s, \phi_{k-1}+\vartheta\left(\phi_{k}-\phi_{k-1}\right), \varepsilon\right) \mathrm{d} \vartheta \mathrm{d} s\right\| \leqq c$.

Further, there exist (by (1.3) and the assumption (II)) continuous non-decreasing positive functions $b_{2}$ and $\omega$ (these functions are essentially moduli of continuity of corresponding integrands with respect to $\varepsilon$ or $\mathbf{x}, \varepsilon)$ such that

(1.21) $\left\|\boldsymbol{D}^{-1} \int_{0}^{2 \pi} e_{\mathscr{S} \mathcal{S}}^{(2 \pi)-s)} f\left(s, \phi_{0}, \varepsilon\right) \mathrm{d} s\right\| \leqq b_{2}(\varepsilon)$,

(1.22) $\left\|D^{-1} \int_{0}^{2 \pi} e_{\mathscr{S}_{\mathcal{N}}}^{(2 \pi-s) \boldsymbol{A}} \int_{0}^{1}\left[f_{\mathbf{x}}\left(s, \phi_{k-1}+\vartheta\left(\phi_{k}-\phi_{k-1}\right), \varepsilon\right)-\boldsymbol{f}_{\mathbf{x}}\left(s, \phi_{0}, 0\right)\right] \mathrm{d} \vartheta \mathrm{d} s\right\| \leqq$ $\leqq \omega(\varepsilon+\rho)$,

while

$(1.23 \mathrm{a}, \mathrm{b})$

$$
b_{2}(\delta) \rightarrow 0, \omega(\delta) \rightarrow 0 \text { for } \delta \rightarrow 0 .
$$

Before proceeding further notice that by $\left(1.11_{0}\right)$

$$
c_{k+1, \mathscr{V}}-\boldsymbol{c}_{k, \mathscr{V}}-\mathbf{D}^{-1} \int_{0}^{2 \pi} e_{\mathscr{S} \mathcal{V}}^{(2 \pi-s) A} f_{\mathbf{x}}\left(s, \phi_{0}, 0\right) e_{\mathscr{V} \mathcal{V}}^{s \boldsymbol{A}}\left(\boldsymbol{c}_{k+1, \mathscr{V}}-\boldsymbol{c}_{k, \mathscr{V}}\right) \mathrm{d} s=0 .
$$

By (1.12), (1.14), (1.21), (1.13) and (1.15)

(1.25) $\left\|c_{1}-c_{0}\right\| \leqq\left\|c_{1, \bar{V}}-c_{0, \bar{r}}\right\|+\left\|c_{1, \mathscr{V}}-c_{0, \mathscr{V}}\right\| \leqq \varepsilon b_{1}+b_{2}(\varepsilon)=B_{1}(\varepsilon)$,

(1.26) $\left\|\phi_{1}-\phi_{0}\right\| \leqq a\left(\varepsilon b_{1}+b_{2}(\varepsilon)\right)+\varepsilon b_{0}=\Phi_{1}(\varepsilon)$. 
By (1.17), (1.12), (1.24), (1.18), (1.13) and (1.20)

(1.27) $\left\|c_{2}-c_{1}\right\| \leqq \varepsilon c_{1}\left\|\phi_{1}-\phi_{0}\right\|+\| c_{1, \mathscr{V}}-c_{0, \mathscr{V}}-D^{-1} \int_{0}^{2 \pi} e_{\mathscr{S} \mathcal{V}}^{(2 \pi-s) A} f_{x}\left(s, \phi_{0}, \varepsilon\right)$. $\cdot\left[e_{\mathscr{N} \mathscr{V}}^{s \boldsymbol{A}}\left(\boldsymbol{c}_{1, \mathscr{V}}-\boldsymbol{c}_{0, \mathscr{V}}\right)+e_{\mathscr{N} \boldsymbol{V}}^{s A}\left(\boldsymbol{c}_{1, \bar{V}}-\boldsymbol{c}_{0, \overline{\mathscr{V}}}\right)+\varepsilon \int_{0}^{s} e^{(s-\sigma) A} f\left(\sigma, \phi_{0}, \varepsilon\right) \mathrm{d} \sigma\right] \mathrm{d} s \|+$ $+\| D^{-1} \int_{0}^{2 \pi} e_{\mathscr{S} \mathcal{N}}^{(2 \pi-s) \boldsymbol{A}} \int_{0}^{1}\left[f_{\mathbf{x}}\left(s, \phi_{0}+\vartheta\left(\phi_{1}-\phi_{0}\right), \varepsilon\right)-f_{\mathbf{x}}\left(s, \phi_{0}, 0\right)\right] \mathrm{d} \vartheta$.

. $\left(\phi_{1}-\phi_{0}\right) \mathrm{d} s\left\|\leqq \varepsilon c_{1}\right\| \phi_{1}-\phi_{0}\left\|+\varepsilon c_{0}+\omega(\varepsilon+\rho)\right\| \phi_{1}-\phi_{0} \|$,

(1.28) $\left\|\phi_{2}-\phi_{1}\right\| \leqq a\left\|c_{2}-c_{1}\right\|+\varepsilon \| \int_{0}^{t} e^{(t-s) A} \int_{0}^{1} f_{x}\left(s, \phi_{0}+\vartheta\left(\phi_{1}-\phi_{0}\right), \varepsilon\right) \mathrm{d} \vartheta$.

. $\left(\phi_{1}-\phi_{0}\right) \mathrm{d} s\left\|\leqq\left(\varepsilon a c_{1}+a \omega(\varepsilon+\rho)+\varepsilon c\right)\right\| \phi_{1}-\phi_{0} \|+\varepsilon a c_{0}$.

By (1.17), (1.24), (1.19) and (1.20) for $k \geqq 2$

$$
\begin{aligned}
& \left\|c_{k+1}-c_{k}\right\| \leqq \\
& \leqq \varepsilon\left\|C \int_{0}^{2 \pi} e_{\overline{\mathscr{S}} \mathcal{S}}^{(2 \pi-s) \mathrm{A}} \int_{0}^{1} f_{x}\left(s, \phi_{k-1}+\vartheta\left(\phi_{k}-\phi_{k-1}\right), \varepsilon\right) \mathrm{d} \vartheta\left(\phi_{k}-\phi_{k-1}\right) \mathrm{d} s\right\|+ \\
& +\| c_{k, \mathscr{V}}-c_{k-1, \mathscr{V}}-D^{-1} \int_{0}^{2 \pi} e_{\mathscr{S} \mathscr{N}}^{(2 \pi-s) A} \mathrm{f}\left(s, \phi_{0}, 0\right)\left[e_{\mathscr{N} \mathfrak{V}}^{s A}\left(c_{k, \mathscr{V}}-c_{k-1, \mathscr{V}}\right) \dot{+}\right. \\
& \dot{+} e_{\mathcal{N}}^{s A}(-1) C \int_{0}^{2 \pi} e_{\overline{\mathscr{V}} \mathcal{N}}^{(2 \pi-s) A} \int_{0}^{1} f_{\mathbf{x}}\left(s, \phi_{k-2}+\right. \\
& \left.+\vartheta\left(\phi_{k-1}-\phi_{k-2}\right), \varepsilon\right) \mathrm{d} \vartheta\left(\phi_{k-1}-\phi_{k-2}\right)+\varepsilon \int_{0}^{s} e^{(s-\sigma) A} \int_{0}^{1} f_{x}\left(\sigma, \phi_{k-2}+\right. \\
& \left.\left.+\vartheta\left(\phi_{k-1}-\phi_{k-2}\right), \varepsilon\right) \mathrm{d} \vartheta\left(\phi_{k-1}-\phi_{k-2}\right)\right] \mathrm{d} s \|+ \\
& +\| D^{-1} \int_{0}^{2 \pi} e_{\mathscr{S} \mathscr{N}}^{(2 \pi \mid s) A} \int_{0}^{1}\left[f_{x}\left(s, \phi_{k-2}+\vartheta\left(\phi_{k-1}-\phi_{k-2}\right), \varepsilon\right)-\right. \\
& \left.-f_{x}\left(s, \phi_{0}, 0\right)\right] \mathrm{d} \vartheta\left(\phi_{k}-\phi_{k-1}\right) \mathrm{d} s \| \leqq \\
& \leqq\left(\varepsilon c_{1}+\omega(\varepsilon+\rho)\right)\left\|\phi_{k}-\phi_{k-1}\right\|+\varepsilon c_{2}\left\|\phi_{k-1}-\phi_{k-2}\right\| \text {, } \\
& \left\|\phi_{k+1}-\phi_{k}\right\| \leqq a\left\|c_{k+1}-c_{k}\right\|+\varepsilon c\left\|\phi_{k}-\phi_{k-1}\right\| \leqq \\
& \leqq\left(\varepsilon a c_{1}+a \omega(\varepsilon+\rho)+\varepsilon c\right)\left\|\phi_{k}-\phi_{k-1}\right\|+\varepsilon a c_{2}\left\|\phi_{k-1}-\phi_{k-2}\right\| \text {. }
\end{aligned}
$$

Let us denote

$$
\begin{aligned}
& \alpha_{0}=\varepsilon c_{2}, \beta_{0}=\varepsilon c_{1}+\omega(\varepsilon+\rho), \\
& \alpha=\varepsilon a c_{2}, \beta=\varepsilon\left(a c_{1}+c\right)+a \omega(\varepsilon+\rho) .
\end{aligned}
$$

It can easily be shown that a series $\sum_{k=0}^{\infty} a_{k}$, where $a_{0}>0, a_{1}>0, a_{k+1}=p a_{k}+$ $+q a_{k-1}, p>0, q>0, k=1,2, \ldots$, is convergent if and only if $p+q<1$. Hence, by (1.20), (1.32) the series $\sum_{k=0}^{\infty}\left\|\phi_{k+1}-\phi_{k}\right\|$ surely converges if $\alpha+\beta<1$. By (1.29) the series $\sum_{k=0}^{\infty}\left\|c_{k+1}-c_{k}\right\|$ then converges, too.

Let us therefore choose a number $q, 0<q<1$ and numbers $\varepsilon_{1}, 0<\varepsilon_{1} \leqq \bar{\varepsilon}_{0}$ and $\rho_{1}, 0<\rho_{1} \leqq \rho_{0}$ such that

$$
\begin{aligned}
& \alpha+\beta=\varepsilon\left(a c_{1}+a c_{2}+c\right)+a \omega(\varepsilon+\rho) \leqq q \text { for } 0 \leqq \varepsilon \leqq \varepsilon_{1} \leqq \varepsilon_{0}, \\
& 0 \leqq \rho \leqq \rho_{1} .
\end{aligned}
$$

By (1.23b) such numbers surely exist. 
Let us now examine in more detail what relations numbers $\varepsilon, \mu$ and $\rho$ must fulfil in order that all solutions of (1.1) beginning in $\mathfrak{l}_{\mu}$ lie for $0 \leqq t \leqq 2 \pi$ in $\mathfrak{X}_{\rho}$. By $\left(1.12_{3}\right)$

$$
\phi_{k}-\phi_{0}=e^{t A}\left(c_{k}-c_{0}\right)+\varepsilon \int_{0}^{t} e^{(t-s) A} f\left(s, \phi_{k-1}, \varepsilon\right) \mathrm{d} s
$$

so that by $(1,16)$

$$
\left\|\phi_{k}-\phi_{0}\right\| \leqq a\left\|c_{k}-c_{0}\right\|+\varepsilon b .
$$

It follows that all $\phi_{k}$ and therefore also $\lim \phi_{k}$ lie in $\mathfrak{X}_{\rho}$ if $\varepsilon$ and $\mu$ are chosen so that

$$
a \mu+b \varepsilon \leqq \rho .
$$

Let us now choose numbers $\varepsilon_{2}>0$ and $\mu_{1}, 0<\mu_{1} \leqq \mu_{0}$ such that

$$
a \mu+b \varepsilon \leqq \rho_{1} \text { for } 0 \leqq \varepsilon \leqq \varepsilon_{2} \text { and } 0 \leqq \mu \leqq \mu_{1} \leqq \mu_{0}
$$

Finally, let us restrict values of $\varepsilon$ so that initial vectors $c_{k}(\varepsilon)$ also lie in ${\stackrel{\mathfrak{r}}{\mu_{1}}}_{\text {}}$.

Evidently,

$$
\left\|c_{j}-c_{0}\right\| \leqq \sum_{k=0}^{\infty}\left\|c_{k+1}-c_{k}\right\|, \quad j=1,2, \ldots
$$

Put

$$
\begin{aligned}
& \gamma=\alpha \beta, \quad \delta=\alpha+\beta^{2}, \\
& \gamma_{0}=\alpha_{0} \beta_{0}, \quad \delta_{0}=\alpha_{0}+\beta_{0}^{2}, \\
& \mathbf{M}=\left(\begin{array}{l}
\alpha \beta \\
\gamma \delta
\end{array}\right), \quad \mathbf{M}_{0}=\left(\begin{array}{l}
\alpha_{0} \beta_{0} \\
\gamma_{0} \delta_{0}
\end{array}\right) .
\end{aligned}
$$

By (1.29) and (1.30)

$$
\begin{aligned}
& \left(\begin{array}{c}
\left\|c_{2 k+1}-c_{2 k}\right\| \\
\left\|c_{2 k+2}-c_{2 k+1}\right\|
\end{array}\right) \leqq M_{0}\left(\begin{array}{c}
\left\|\phi_{2 k-1}-\phi_{2 k-2}\right\| \\
\left\|\phi_{2 k}-\phi_{2 k-1}\right\|
\end{array}\right), \\
& \left(\begin{array}{l}
\left\|\phi_{2 k+1}-\phi_{2 k}\right\| \\
\left\|\phi_{2 k+2}-\phi_{2 k+1}\right\|
\end{array}\right) \leqq M\left(\begin{array}{c}
\left\|\phi_{2 k-1}-\phi_{2 k-2}\right\| \\
\left\|\phi_{2 k}-\phi_{2 k-1}\right\|
\end{array}\right) .
\end{aligned}
$$

Hence

$$
\begin{gathered}
\sum_{k=0}^{\infty}\left\|c_{k+1}-c_{k}\right\| \leqq \\
\left(\left\|c_{1}-c_{0}\right\|+\left\|c_{2}-c_{1}\right\|+\left\|M_{0}\right\|(1-\|M\|)^{-1} .\right. \\
\left(\left\|\phi_{1}-\phi_{0}\right\|+\left\|\phi_{2}-\phi_{1}\right\|\right) .
\end{gathered}
$$

From (1.37a) and from $\alpha<1$ it follows that

$$
\|\mathbf{M}(\varepsilon, \rho)\|=\max (\alpha+\gamma, \beta+\delta)=\beta+\delta=\beta(\varepsilon, \rho)+\delta(\varepsilon, \rho),
$$

for $\alpha+\gamma=\alpha+\alpha \beta<\beta+\delta=\beta+\alpha+\beta^{2}$ and similarly

$$
\left\|\mathbf{M}_{0}(\varepsilon, \rho)\right\|=\max \left(\alpha_{0}+\gamma_{0}, \beta_{0}+\delta_{0}\right)=\beta_{0}+\delta_{0}=\alpha_{0}+\beta_{0}\left(1+\beta_{0}\right) \text {. }
$$


By (1.36), (1.41), (1.42), (1.43) and (1.25)-(1.28) all $c_{k}$ (and therefore $\lim _{k \rightarrow \infty} c_{k}$, too) will lie in $\mathfrak{R}_{\mu_{1}}$, if we choose $\varepsilon_{3}>0$ such that

$$
\begin{aligned}
& B_{1}(\varepsilon)+\left[\varepsilon_{0} c_{1}+\omega\left(\varepsilon_{0}+\rho_{1}\right)\right] \Phi_{1}(\varepsilon)+\varepsilon c_{0}+\left\|\boldsymbol{M}\left(\varepsilon_{0}, \rho_{1}\right)\right\|\left(1-\| \boldsymbol{M}\left(\varepsilon_{0}, \rho_{1}\right)\right)^{-1} . \\
& \cdot\left\{\Phi_{1}(\varepsilon)+\left[\varepsilon_{0}\left(a c_{1}+c\right)+a \omega\left(\| \varepsilon+\rho_{1}\right)\right] \Phi_{1}(\varepsilon)+\varepsilon a c_{0}\right\} \leqq \mu_{1} \text { for } 0 \leqq \varepsilon \leqq \varepsilon_{3} .
\end{aligned}
$$

Such $\varepsilon_{3}$ really exists because of (1.23a). Put

$$
\varepsilon^{*}=\min \left(\varepsilon_{1}, \varepsilon_{2}, \varepsilon_{3}\right), \mu^{*}=\mu_{1}, \rho^{*}=\rho_{1} .
$$

Define functions $c^{*}(\varepsilon)$ and $\phi^{*}\left(t, c^{*}(\varepsilon), \varepsilon\right)$ as

$$
\begin{gathered}
c^{*}(\varepsilon)=\lim _{k \rightarrow \infty} c_{k}=c_{0}+\sum_{k=0}^{\infty}\left(c_{k+1}-c_{k}\right), \\
\phi^{*}\left(t, c^{*}(\varepsilon), \varepsilon\right)=\lim _{k \rightarrow \infty} \phi_{k}(t)=\phi_{0}(t)+\sum_{k=0}^{\infty}\left(\phi_{k+1}(t)-\phi_{k}(t)\right) .
\end{gathered}
$$

For $0 \leqq \varepsilon \leqq \varepsilon^{*}$, all solutions $\phi(t, c, \varepsilon)$ of $(1.1)$ with initial conditions $\phi(0, c, \varepsilon)=$ $=c \in \mathfrak{R}_{\mu^{*}}$ remain for $0 \leqq t \leqq 2 \pi$ in $\mathfrak{X}_{\rho^{*}} \subset \mathfrak{X}_{\rho_{0}}$, so that assumptions $\left(P_{1}\right)-\left(P_{3}\right)$ under which our estimations were derived are fulfilled.

Hence by former considerations the limit (1.46) exists uniformly and absolutely with respect to $\varepsilon$ for $0 \leqq \varepsilon \leqq \varepsilon^{*}$ and the limit (1.47) exists uniformly and absolutely with respect to $t$ and $\varepsilon$ for $0 \leqq t \leqq 2 \pi$ and $0 \leqq \varepsilon \leqq \varepsilon^{*}$.

Thus, in systems $\left(1.12_{1,2,3}\right)$ we can let $k \rightarrow \infty$. under the integral signs. Hence it follows that the functions $c^{*}(\varepsilon)$ and $\phi\left(t, c^{*}(\varepsilon), \varepsilon\right)$ fulfil the systems of equations (1.6), (1.9) and (1.10). These systems are equivalent to (1.1), (1.9) and (1.10). This means that the function $\phi^{*}\left(t, c^{*}(\varepsilon), \varepsilon\right)$ is a solution of (1.1) and fulfils the periodic boundary conditions $\phi^{*}\left(2 \pi, c^{*}(\varepsilon), \varepsilon\right)=\phi^{*}\left(0, c^{*}(\varepsilon), \varepsilon\right)=c^{*}(\varepsilon)$, q. e. d.

The estimation of $\varepsilon^{*}$ can be simplified by supposing that the function $f(t, \mathbf{x}, \varepsilon)$ is of class $C^{1 L}$ in $\mathbf{x}$ and of class $C^{0 L}$ in $\varepsilon$ on $\mathfrak{M}$. Since a modulus of continuity $\omega(\delta)$ of a function satisfying a Lipschitz condition is of the form $\omega(\delta)=K \delta$ we can put in (1.21) and (1.22)

$$
b_{2}(\varepsilon)=\varepsilon \tilde{b}_{2}, \quad \omega(\varepsilon+\rho)=(\varepsilon+\rho) \tilde{\omega},
$$

where $\tilde{b}_{2}$ and $\tilde{\omega}$ are constants. Put

$$
\begin{gathered}
A=a\left(c_{1}+c_{2}+\tilde{\omega}\right)+c \\
B=b_{1}+\tilde{b}_{2}+\left(\varepsilon_{0} c_{1}+\left(\varepsilon_{0}+\rho_{0}\right) \tilde{\omega}\right)\left[a\left(b_{1}+\tilde{b}_{2}\right)+b_{0}\right]+c_{0}+ \\
+\left\|\boldsymbol{M}_{0}\left(\varepsilon_{0}, \rho_{0}\right)\right\|\left(1-\mathbf{M}\left(\varepsilon_{0}, \rho_{0} \|\right)^{-1}\left\{\left[a\left(b_{1}+b_{2}\right)+b_{0}\right] .\right.\right. \\
\left.\quad \cdot\left[1+\varepsilon_{0}\left(a c_{1}+c\right)+a\left(\varepsilon_{0}+\rho_{0}\right) \tilde{\omega}+\varepsilon_{0} a c_{0}\right]\right\} .
\end{gathered}
$$

By (1.33), (1.35) and (1.44) we have to choose positive constants $q<1, \varepsilon_{1}, \rho_{1}, \varepsilon_{2}, \mu_{1}$ and $\varepsilon_{3}$ such that

$$
\begin{gathered}
A \varepsilon_{1}+\tilde{\omega} \rho_{1} \leqq q<1, \\
a \mu_{1}+b \varepsilon_{2} \leqq \rho_{1}, \\
B \varepsilon_{3} \leqq \mu_{1} .
\end{gathered}
$$


The inequalities will be fulfilled (in the most favourable way) if we put

$$
\begin{aligned}
& \tilde{\varepsilon}^{*}=\varepsilon_{1}=\varepsilon_{2}=\varepsilon_{3}=\frac{q}{A+\tilde{\omega}(b+a B)}, \\
& \tilde{\mu}^{*}=\min \left(\frac{B q}{A+\tilde{\omega}(b+a B)}, \mu_{0}\right), \quad \tilde{\rho}^{*}=\min \left(\frac{(b+a B) q}{A+\tilde{\omega}(b+a B)}, \rho_{0}\right) .
\end{aligned}
$$

Hence, we may state the following theorem.

Theorem 1.2. Let the following assumptions be fulfilled.

(I) and (III) the same as in Theorem 1.1.

(II) The function $\mathbf{f}(t, \mathbf{x}, \varepsilon)$ is of class $C^{0}$ in $t$, of class $C^{1 L}$ in $\mathbf{x}$ and of class $C^{0 L}$ in $\varepsilon$ on $\mathfrak{M}$.

(IV) $\tilde{\varepsilon}^{*}, \tilde{\mu}^{*}$ and $\tilde{\rho}^{*}$ are defined by $\left(1.45^{\prime}\right)$.

Then for every $\varepsilon$ from the interval $\left(0, \tilde{\varepsilon}^{*}>\right.$ there exists a unique $2 \pi$-periodic solution $\boldsymbol{\phi}^{*}\left(t, \mathbf{c}^{*}(\varepsilon), \varepsilon\right)$ of the equation (1.1) while $\left\|\mathbf{c}^{*}(\varepsilon)-\mathbf{c}^{(0) *}\right\| \leqq \tilde{\mu}^{*}$ and $\| \boldsymbol{\phi}^{*}\left(t, \mathbf{c}^{*}(\varepsilon), \varepsilon\right)-$ $-e^{t \boldsymbol{A}} c^{(0) *} \| \leqq \tilde{\rho}^{*}$ for $0 \leqq t \leqq 2 \pi$.

Remark 1. Clearly, if we were supposing that $f(t, x, \varepsilon)$ is defined and of class $C^{0}$ in $\varepsilon$ for $\left(-\varepsilon_{0}, \varepsilon_{0}\right)$ instead of $\left(0, \varepsilon_{0}\right)$ we could prove that a unique $2 \pi$-periodic solution of (1.1) exists for $\varepsilon \in\left\langle-\varepsilon^{*}, \varepsilon^{*}\right\rangle, \varepsilon \neq 0, \varepsilon^{*}$ having the same meaning as in Th. 1.1.

Remark 2. By omitting some evident terms, the former estimations could be used in the nonresonant case, too. But in this case we can get under less restrictive assumptions a simpler estimation.

Theorem 1.3. Let the following assumptions be fulfilled.

(I) The matrix A has no characteristic root of the type pi ( $p$ being an integer including 0$)$.

(II) The function $\boldsymbol{f}(t, \mathbf{x}, \varepsilon)$ is on $\mathfrak{M}=E\left[t, \boldsymbol{x}, \varepsilon \mid, \quad 0 \leqq t \leqq 2 \pi, \quad\|\mathbf{x}\|<\rho_{0}, 0<\right.$ $\left.<\varepsilon<\varepsilon_{0}\right] 2 \pi$-periodic and of class $C^{0}$ in $t$, of class $C^{0 L}$ in $\mathbf{x}$ and of class $C^{0}$ in $\varepsilon$.

(III) Let us denote $L_{1}$ and $L_{2}$ constants (whose existence is guaranteed by (II)) satisfying inequalities

$$
\begin{aligned}
& \left.\| e^{2 \pi \boldsymbol{A}}-\boldsymbol{E}\right)^{-1} \int_{0}^{2 \pi} e^{(2 \pi-s) \boldsymbol{A}}\left[\boldsymbol{f}\left(s, \boldsymbol{\phi}^{\prime}, \varepsilon\right)-\boldsymbol{f}\left(s, \boldsymbol{\phi}^{\prime \prime}, \varepsilon\right)\right] \mathrm{d} s\left\|\leqq L_{1}\right\| \boldsymbol{\phi}^{\prime}-\boldsymbol{\phi}^{\prime \prime} \|, \\
& \left\|\int_{0}^{t} e^{(t-s) \boldsymbol{A}}\left[\boldsymbol{f}\left(s, \boldsymbol{\phi}^{\prime}, \varepsilon\right)-\boldsymbol{f}\left(s, \boldsymbol{\phi}^{\prime \prime}, \varepsilon\right)\right] \mathrm{d} s\right\| \leqq L_{2}\left\|\boldsymbol{\phi}^{\prime}-\boldsymbol{\phi}^{\prime \prime}\right\| .
\end{aligned}
$$

Let $\varepsilon^{*}=\min \left(\varepsilon_{0}, \varepsilon_{1}\right), \mu^{*}=\mu_{1}$ where $\varepsilon_{1}>0$ and $\mu_{1}>0$ are numbers satisfying inequalities

$$
\begin{gathered}
\varepsilon_{1}\left(a L_{1}+L_{2}\right) \leqq q<1, a \mu_{1}+b \varepsilon_{1} \leqq \rho_{0}, \\
\varepsilon_{1}\left[b_{1}+\frac{\varepsilon_{1} L_{1}}{1-\varepsilon_{1}\left(a L_{1}+L_{2}\right)}\left(a b_{1}+b_{0}\right)\right] \leqq \mu_{1},
\end{gathered}
$$

where $a, b, b_{0}$ and $b_{1}$ have the same meaning as in Th. 1.1 (of course, it is now $\overline{\mathscr{S}}=$ $=\overline{\mathscr{V}}=\mathscr{N})$. 
Then for every $\varepsilon$ from the interval $\left(0, \varepsilon^{*}\right\rangle$ there exists a unique $2 \pi$-periodic solution $\phi\left(t, \mathbf{c}^{*}(\varepsilon), \varepsilon\right)$ of the equation (1.1) with initial value $\phi\left(0, \boldsymbol{c}^{*}(\varepsilon), \varepsilon\right)=\boldsymbol{c}^{*}(\varepsilon)$ while $\left\|c^{*}(\varepsilon)\right\| \leqq \mu^{*}$ and $\left\|\phi\left(t, c^{*}(\varepsilon), \varepsilon\right)\right\| \leqq \rho^{*}$.

The proof of this theorem is easy and may be omitted.

\section{AN EXISTENCE THEOREM IN THE LARGE}

Frequently, we are not interested as much in the interval of $\varepsilon$, for whose values a certain approximate method is convergent as in the interval, for whose values a periodic solution $\phi(t, \boldsymbol{c}(\varepsilon), \varepsilon)$ (with the property $\phi(t, \mathbf{c}(\varepsilon), \varepsilon) \rightarrow e^{t A} \boldsymbol{c}^{(0) *}$ for $\varepsilon \rightarrow 0$ ) exists at all. Let us derive a theorem of such global character. (Unfortunately, the utilization of this thoerem is very difficult).

Let us state three definitions (cf. [9], chap. VIII, sec. 3).

Definition 2.1. A sheet of points in $\varepsilon$, c-space is a connected set $W_{0}$ of points $\mathbf{w}=(\varepsilon, \boldsymbol{c})$ with finite coordinates such that for every point $\mathbf{w}_{0}=\left(\varepsilon_{0}, \boldsymbol{c}_{0}\right)$ of $W_{0}$, there exists a neighborhood $N\left(\mathbf{w}_{0} ; a\right)=E\left[\mathbf{w} \mid\left\|\mathbf{w}-\mathbf{w}_{0}\right\|<a\right]$ such that no two points of $W_{0}$ in $N\left(\mathbf{w}_{0} ; a\right)$ have the same projection $\varepsilon_{0}$; and for every $\mathbf{w}_{0}$ in $W_{0}$ and every $a>0$ there is a neighborhood $N\left(\mathbf{w}_{0} ; b\right)$ each of whose points $\varepsilon$ is a projection of a point $\boldsymbol{w}$ of $W_{0}$ in $N\left(\mathbf{w}_{0}, a\right)$.

Definition 2.2. A boundary point of a sheet $W_{0}$ is a point not belonging to $W_{0}$, but every neighborhood of which contains points of $W_{0}$.

Definition 2.3. If a vector function $\boldsymbol{h}(\varepsilon, \boldsymbol{c})$ is defined and of class $C^{1}$ in $\boldsymbol{c}$ for $(\varepsilon, \boldsymbol{c})$ in an open set $W$, then a point $\mathbf{w}=(\varepsilon, \boldsymbol{c})$ is called an ordinary point for $\boldsymbol{h}(\varepsilon, \boldsymbol{c})$ in case that $\mathbf{w} \in W$ and the matrix $\boldsymbol{h}_{\boldsymbol{c}}(\varepsilon, \boldsymbol{c})$ is nonsingular. All other points are called exceptional points.

Let us prove the following theorem:

Theorem 2.1. Given the equation (1.1), let the following assumptions be fulfilled.

(I)-(III) the same as in theorem 1.1 except that in (II) $-\varepsilon_{0}<\varepsilon<\varepsilon_{0}, \varepsilon_{0}>0$.

(IV) For $\varepsilon \in \mathbb{E}=\left(-\varepsilon_{0}, \varepsilon_{0}\right)$ and $c \in \mathfrak{R}, \mathfrak{L}$ being an open connected set, the solutions of (1.1) are uniquely determined and for $0 \leqq t \leqq 2 \pi$ stay in $\mathfrak{X}_{\rho_{0}}$.

Then there exists the unique sheet of solutions $c=c^{*}(\varepsilon)$ of the system

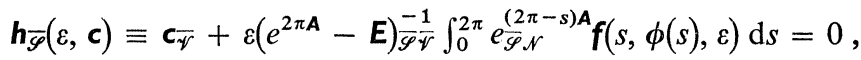

$$
\begin{aligned}
& \boldsymbol{h}_{\mathscr{S}}(\varepsilon, \boldsymbol{c}) \equiv \int_{0}^{2 \pi} e_{\mathscr{Y} \mathcal{H}}^{(2 \pi-s) A} f(s, \phi(s), \varepsilon) \mathrm{d} s=0,
\end{aligned}
$$


where $\phi(t)=\phi(t, c, \varepsilon)$ is the solution of

$$
\mathbf{x}(t)=e^{t \boldsymbol{A}} \boldsymbol{c}+\varepsilon \int_{0}^{t} e^{(t-s) A} f(s, \mathbf{x}(s), \varepsilon) \mathrm{d} s,
$$

with these properties:

(a) $\left(0, c^{(0) *}\right) \in W$,

(b) every point $W$ is an ordinary point;

(c) the only finite boundary points of Ware either boundary points of $\mathfrak{G}=\mathbb{E} \times \mathfrak{l}$ or exceptional points of the function

$$
\boldsymbol{h}(\varepsilon, \mathbf{c})=\boldsymbol{h}_{\overline{\mathscr{A}}}(\varepsilon, \boldsymbol{c})+\boldsymbol{h}_{\mathscr{S}}(\varepsilon, \boldsymbol{c}) .
$$

Proof. By the definition of $(S)$ the system 2.2 has for every $(\varepsilon, \boldsymbol{c}) \in(S)$ a unique solution remaining in $\mathfrak{X}_{\rho_{0}}$ for $0 \leqq t \leqq 2 \pi$. Substitute this solution into (2.1). As long as $(\varepsilon, c) \in(S)$ the left-hand sides of these equations are defined and of class $C^{1}$ in $c$. From this the assertion of the theorem immediately follows by [9], chap. VIII, sec. 3, th. 3 .

\section{Bibliography}

[1] A. A. Круминг: Оценка радиуса сходимости рядов по степеням малого параметра, представляющих периодические решения систем дифференциальных уравнений. Укр. мат. журн. 5 (1953), 434-438.

[2] $A$. A. Круминг: Оценка интервала изменения малого параметра, входящего в правые части системы дифференциальных уравнений, в котором существуют периодические решения. Москва, Учен. зап. Обл. пед. ин-та, 39: Труды кафедры математики, 3 (1956), 61-66. (This paper is not available in Czechoslovakia.)

[3] A. E. Гельман: Один признак существования определенных классов решений нелинейного дифференциального уравнения и некоторые оценки в методе малого параметра. ДАН 118: 6 (1958), $1063-1065$.

[4] Ю. А. Рябов: Оценка области сходимости периодических рядов - решений дифференциальных уравнений с малым параметром. ДАН 118: 4 (1958), 642-645.

[5] D. C. Lewis: Periodic solutions of differential equations containing a parameter. Duke Math. J. $22: 1$ (1955), 39-56.

[6] D. C. Lewis: On the perturbation of a periodic solution when the variational system has nontrivial periodic solutions. J. Rat. Mech. Anal. 4 : 5 (1955), 795-815.

[7] J. Kurzweil: К теории колебаний автономной квазилинейной системы обыкновенных дифференциальных уравнений. Чех. мат. журн. 5 (80) (1955), 517-531.

[8] E. A. Coddington - N. Levinson: Theory of ordinary differential equations. New York, 1955.

[9] L. M. Graves: The theory of functions of real variables. New York, 1956. 


\title{
О ПЕРИОДИЧЕСКИХ РЕШЕНИЯХ КВАЗИЛИНЕЙНОЙ НЕАВТОНОМНОЙ СИСТЕМЫ
}

\author{
ОТТО ВЕЙВОДА (Otto Vejvoda), Прага
}

В работе исследуются условия существования периодических решений с периодом $2 \pi$ системы (1.1). Предполагается, что матрица имеет характеристические корни типа $p i$ ( $p$ - целое число вклучительно нуля) и что функция f - периодическая с периодом $2 \pi$ в переменной $t$.

В теореме 1.1 далее предполагается, что (а) система уравнений (1.2) и (1.3) имеет действительное решение $c^{(0)}=c^{(0) *}$ и что (b) $f(t, x, \varepsilon) \in C^{0,1,0}$ в некоторой окрестности $2 \pi$-периодического решения $e^{t A} c^{(0) *}$ системы (1.4). Указывается число $\varepsilon^{*}$ такое, что если только $0<\varepsilon<\varepsilon^{*}$, то система (1.1) имеет одно и только одно $2 \pi$-периодическое решение, которое для $\varepsilon \rightarrow 0$ стремится к $\mathrm{e}^{t \boldsymbol{A}} \mathrm{c}^{(0) *}$.

В теореме 1.2 оценка числа $\varepsilon^{*}$ несколько улучшается в силу более сильного предположения, что $f(t, x, \varepsilon) \in C^{0,1 L, 0 L}$ (значит, что $f$ имеет частные производные 1-го порядка по $\boldsymbol{x}$, удовлетворяющие условию Липшица).

Теорема 2.1 в сушности гласит, что система (1.1) имеет $2 \pi$-периодическое решение для всех $\varepsilon$, для которых решения системы (1.1) существуют на отрезке $\langle 0,2 \pi\rangle$, если якобиян системы (2.1), где $\phi(s)$ - решение системы (2.2), отличен от нуля. 